\title{
Increased physical fitness after 4-week training on a new hybrid FES-cycle in persons with spinal cord injury
}

\author{
P.J.C. Heesterbeek ${ }^{\mathrm{a}, *}$, H.W.A. Berkelmans ${ }^{\mathrm{b}}$, D.H.J. Thijssen ${ }^{\text {c }}$, H.J.M. van Kuppevelt ${ }^{\mathrm{d}}$, \\ M.T.E. Hopman ${ }^{\mathrm{c}}$ and J. Duysens $\mathrm{s}^{\mathrm{a}, \mathrm{e}}$

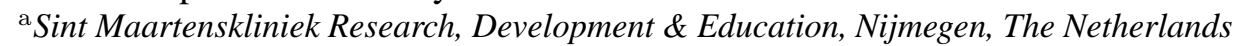 \\ ${ }^{\mathrm{b}}$ BerkelBike, Nijmegen, The Netherlands \\ ${ }^{\mathrm{c}}$ Department of Physiology, Radboud University Nijmegen Medical Centre, Nijmegen, The Netherlands \\ ${ }^{\mathrm{d}}$ Sint Maartenskliniek Rehabilitation Center, Nijmegen, The Netherlands \\ ${ }^{\mathrm{e}}$ Department of Rehabilitation, Radboud University Nijmegen Medical Centre, Nijmegen, The Netherlands
}

\begin{abstract}
The purpose of the present study was to determine the effect of a 4-week training program on a newly developed hybrid functional electrical stimulation (FES)-cycle on physical fitness in spinal cord-injured (SCI) individuals.

Ten SCI individuals (age 23-53 years, lesion level range T3-T11) participated and trained 8-12 times in 4 weeks on the hybrid FES-cycle (voluntary arm-contractions and stimulation of leg muscles). Leg volume was measured. During a graded hybrid exercise test, peak oxygen consumption (VO2peak), peak power output (POpeak) and power of the legs (dP) were measured pre and post training.

Upper leg volume increased significantly $(8.5 \%(p=0.047)$ and $8.3 \%(p=0.018)$ for the right and left leg, respectively). POpeak and VO2peak increased $11.7 \%(p=0.012)$ and $9.3 \%(p=0.015)$, respectively. There was no significant difference in $\mathrm{dP}$ between pre and post training. After only 4 weeks of training, considerable training effects were observed, which are comparable to longer training studies in literature. The results of this study indicate that hybrid training on the new hybrid FES-cycle is an appropriate training method for individuals with a spinal cord injury to increase physical fitness.
\end{abstract}

Keywords: Paraplegia, functional electrical stimulation, outdoor hybrid FES-cycle, graded hybrid exercise test, physical fitness

\section{Introduction}

Following spinal cord injury (SCI), the body undergoes marked changes such as loss of motor control, sensibility and autonomic function below the level of the lesion. In addition to a decrease in cardiovascular condition (including deficient cardiovascular reflex responses and inactivity of the muscle pump), this chronic form of muscular inactivity leads to marked

*Address for correspondence: Petra Heesterbeek, Sint Maartenskliniek Research, Development \& Education, Postbox 9011, 6500 GM Nijmegen, The Netherlands. Tel.: +31 24 3659628; Fax: +31 24 3659154; E-mail: P.Heesterbeek@ maartenskliniek.nl. changes in muscles and peripheral circulation $[11,13$, 14,23]. Characteristic changes such as muscle disuse atrophy and a reduction of oxidative capacity appear [4]. In addition there is a shift in muscle fiber composition from type I (slow-oxidative) to type II fibers (fast-glycolytic) [29]. The peripheral circulation deteriorates due to atrophy of the vascular system below the lesion and there is an increasing incidence of osteoporosis and pressure sores [19]. These progressive problems may eventually influence the rehabilitation process and limit the functionality in daily life [13].

Rehabilitation programs and sports activities performed with the muscles of the upper body alone are not sufficient to reduce the additional problems caused 
by SCI [8]. For individuals with SCI it is difficult to train the cardiovascular system using arm-activity alone, because the aerobic capacity is limited more by peripheral factors, such as a decreased active muscle mass and venous blood pooling, than by central cardiac factors $[2,17]$. Therefore a method called functional electrical stimulation-induced leg cycle ergometry (FES-LCE) was developed in the 1980s as a supplementary method to help prevent or reduce the health problems mentioned above, by increasing the amount of active muscle mass during training [14,23]. With FES three paralyzed leg muscle(group)s (the quadriceps, hamstrings and gluteal muscles) are electrically activated and when applied on a leg cycle ergometer, one has a method to achieve a better cardiovascular fitness for the patients. Several studies have indicated beneficial effects of FES-LCE for individuals with SCI. Prolonged electrical stimulation can achieve increased muscle mass $[1,9,26]$, increased oxidative capacity of the muscle [5], increased peripheral blood flow $[6,8,9]$, increased range of motion (ROM) of the joints, decreased osteoporosis and decreased incidence of spasms [27,29]. These changes result in a reduced risk of secondary medical complications such as pressure sores $[13,14,19]$. FES-LCE also increases mental fitness due to hormonal changes resulting from exercise [16]. Furthermore, several authors reported an improved aerobic capacity (increased peak oxygen uptake) with FES-training due to central (i.e. an increased stroke volume) and peripheral adaptations (i.e. a conversion towards more oxidative type I fibers) [5]. FES cycling provides individuals with SCI a method to train at a sufficient intensity to obtain marked cardiopulmonary adaptations $[8,19]$.

Several authors concluded that hybrid training (FESLCE or FES isometric work and voluntary arm work) is a better training method than either FES-LCE or arm cranking exercise alone $[3,18,25,30]$. With hybrid exercise a substantially larger muscle mass is utilized and the venous return is enhanced [10]. The methods used for hybrid cycling mostly required laboratory settings, where an arm crank ergometer was placed over a FEScycle ergometer $[18,25]$. In addition to the mechanical disadvantages of this experimental setting, the subjects had to come over to the laboratory for (hybrid) training. There were several attempts for constructing outdoor FES vehicles for independent usage, but until now they all failed on functionality [7,20-22,31]. Therefore, a new hybrid FES-cycle was developed, allowing FEScycling outdoors. It uses FES stimulation to drive the leg movements while voluntary arm contractions are needed to produce arm cranking, which is often required to assist propulsion. In addition to diminishing secondary medical complications by enhancing physical fitness, this hybrid FES-cycle promises improvement of mobilization of the SCI population.

The purpose of this study is to investigate the effect of 4-week training on a hybrid FES-cycle on the physical fitness of spinal cord-injured individuals.

\section{2. $\operatorname{Method}(\mathbf{s})$}

\subsection{Study design}

This study describes the first functional tests with this new hybrid FES-cycle and is set up as a pilot study. The study consisted of a 4-week training period. To evaluate the training period a series of measurements was performed before and after training.

\subsection{Subjects}

Ten volunteers, 9 male and 1 female, with paraplegia participated in this study. Mean age of the subjects was 39.3 (8.7) years, mean height and mean body mass were $1.82(0.052) \mathrm{m}$ and 73.1 (12.6) kg, respectively. Lesion levels ranged from Th2 to Th12, 9 subjects were classified as ASIA A, 1 as ASIA C. Mean time since injury was 10.5 (6.4) years. All subjects were experienced hand bike users, had complete arm function and did not participate in FES training at least 1 year prior to the study. Prior to participation, subjects were screened by an independent physician and underwent an ECG recording to investigate contra-indications for exercise and FES. The local medical ethics committee (region Nijmegen) approved this study. A written informed consent was obtained from all subjects prior to participation.

\subsection{Hybrid FES-cycle}

The newly developed hybrid FES-cycle is a combination of a recumbent bike and a hand bike. It consists of a front part, which is a one-wheeled bike unit, and a common wheelchair. The wheel of the front part is arm and leg power driven. With an adapter under the wheelchair, the user can couple and uncouple the front part while seated in his wheelchair. The principle of this hybrid FES-cycle is patented. For a schematic overview of the front part of the FES-cycle, see Fig. 1. 


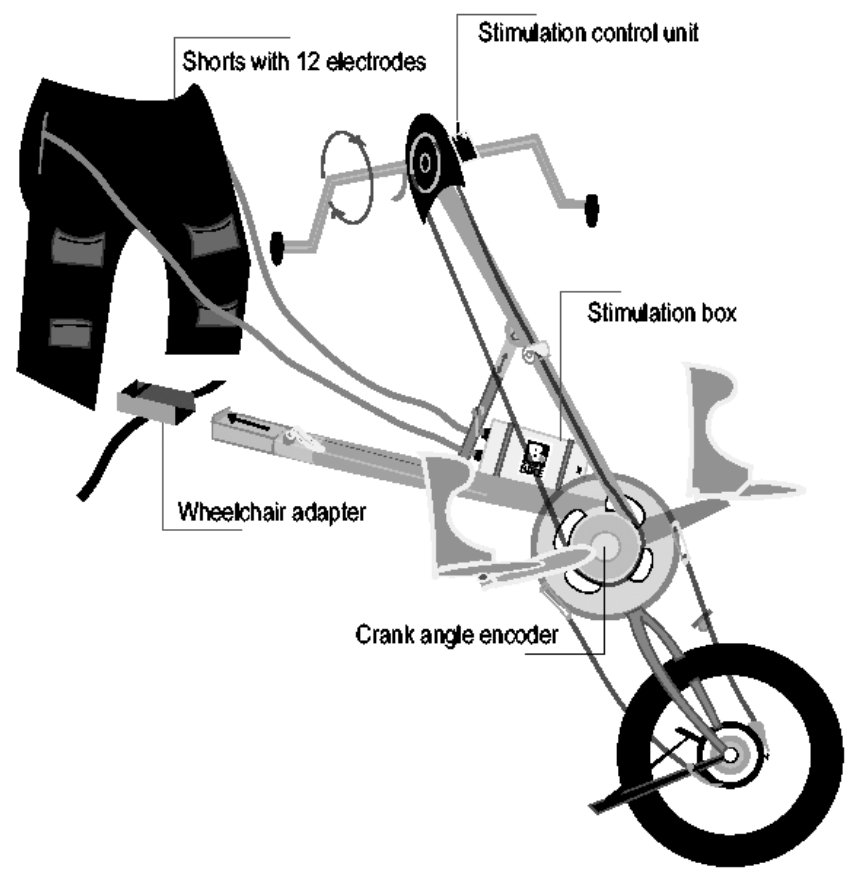

Fig. 1. Schematic overview of the hybrid FES-cycle. This front part can be attached to the user's wheelchair by the wheelchair adapter. The cycle is propelled by stimulated leg muscles and voluntary arm work. The user regulates muscular stimulation by the stimulation control unit, that is connected to the stimulation box. This box gets input from the crank angle encoder that controls timing of the muscular stimulation by monitoring crank position. The stimulation box stimulates the muscles through the electrodes in the shorts.

The hybrid FES-cycle has a FES unit. This consists of a small box $(18 \times 11 \times 6 \mathrm{~cm})$ that is connected to an incremental angle encoder, brake sensor and an amplitude control unit. The encoder is placed over the crank axle and gives the position of the crank in 56 units; this information is pre-requisite for adequate onset of muscle stimulation. The brake sensor interrupts the stimulation when the handbrake is used. The amplitude of the stimulation is controlled by a switch, positioned on the arm crank, which allows the user to select one of 16 levels ( $9 \mathrm{~mA}$ per level). The maximum stimulation intensity is $145 \mathrm{~mA}$ with a frequency of $35 \mathrm{~Hz}$. A biphasic square pulse shape with a total pulse time of $1 \mathrm{~ms}$ has been chosen. The timing of the stimulation of the muscles is adjusted by the FES unit and is based on the cadence.

In this study we used the hybrid FES-cycle in a laboratory setting. However, the hybrid FES-cycle can be used outdoors using a pair of shorts with 6 pairs of electrodes (Bioflex, Columbus, Ohio), which stimulate the quadriceps, hamstrings and gluteus muscles of each leg. After connecting the front part to the wheelchair, the legs were placed in customized carbon leg supporters to correct for unwanted side movements of the knee.

\subsection{Training}

Subjects trained 8-12 times within 4 weeks at the rehabilitation center. Each training session consisted of a 5-minute warming-up (voluntary arm exercise), 30 minutes of hybrid cycling (voluntary arm and FES leg exercise) and a 5-minute cooling-down (arm exercise). All subjects trained on the hybrid FES-cycle on a roller (Tacx, Wassenaar, The Netherlands). The experimenter manually adjusted the stimulation intensity based on decreased power output indicating fatigue of the stimulated muscle groups. During this training period, adhesive surface electrodes (Farmadomo, Nuland, The Netherlands) were placed bilaterally over the neuromuscular motor points of the quadriceps, hamstrings and gluteus muscles. Subjects trained at $60-70 \%$ of their estimated peak oxygen consumption. During all sessions a Polar sport tester recorded the heart rate every 60 seconds, to determine the objective training intensity.

\subsection{Measurements}

\subsubsection{Leg volume}

Leg volume measurements were performed of both legs in supine position by measuring circumferences 


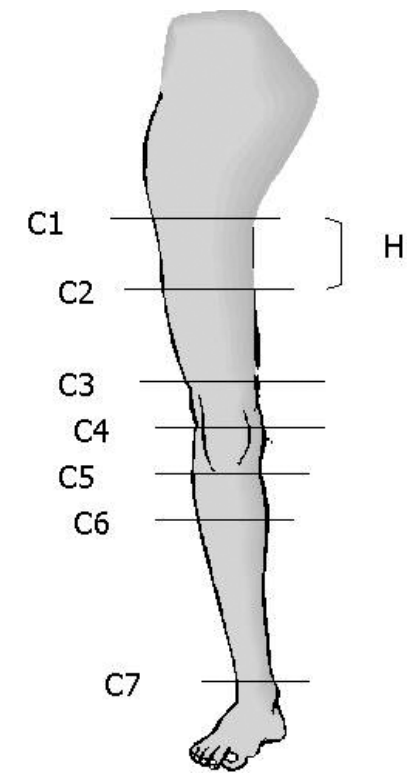

Fig. 2. Methods for calculating leg volume. Each part of the leg is approached as a cone. The volume of each cone is calculated using the formula: Volume cone $(L)=\frac{1}{12 \pi} \cdot H \cdot\left(C_{\text {upper }^{2}}+\left(C_{\text {upper }}\right.\right.$. $\left.C_{\text {lower }}\right)+C_{\text {lower }}$ ). $\mathrm{C} 1=$ upper leg, C2 is measured at $1 / 3$ of distance $\mathrm{C} 1-\mathrm{C} 4, \mathrm{C} 3=$ supra patellar, $\mathrm{C} 4=$ mid patellar, $\mathrm{C} 5=$ sub patellar, $\mathrm{C} 6=$ maximum calf, $\mathrm{C} 7=$ minimal ankle. Upper leg volume $=$ upper three cones from $\mathrm{C} 1$ to $\mathrm{C} 4$, lower leg volume=lower three cones from $\mathrm{C} 4$ to $\mathrm{C} 7$. $\mathrm{H}=$ height between adjacent circumferences.

(C) and heights (H) in cm (Fig. 2). The leg is divided into six imaginary cones. For each cone the volume was calculated using the formula:

$$
\begin{aligned}
\text { Volume cone }(L)= & \frac{1}{12 \pi} \cdot H \cdot\left(C_{\text {upper }^{2}}+\right. \\
& \left.\left(C_{\text {upper }} \cdot C_{\text {lower }}\right)+C_{\text {lower }^{2}}\right)
\end{aligned}
$$

according to Jones et al. (1969) [15].

Upper and lower leg volume were calculated. To control for increase in leg volume caused by increase in body fat, skin-fold thickness at the quadriceps muscle was measured (Slimguide, Bodycare, Kenilworth, United Kingdom).

\subsubsection{Graded hybrid exercise test (GHT)}

To evaluate physical fitness, subjects performed a graded hybrid exercise test (GHT) on the hybrid FEScycle. This GHT consisted of an initial warming-up phase at $10 \mathrm{~W}$ for 3 minutes. Directly following this warming-up the actual test started with a 3-minute sub maximal stage at $20 \mathrm{~W}$, followed by an increase in workload of $10 \mathrm{~W}$ every minute and $20 \mathrm{~W}$ after $16 \mathrm{~min}$ utes. Subjects were instructed to maintain pedal cadence at 50 rotations per minute (RPM) and were ver- bally encouraged. Subjects performed the test while using their arms (voluntary arm cranking) and legs (FES cycling) simultaneously. The electrical stimulation was increased manually during the test. Prior to the first test the subject's resting heart rate and peak heart rate were estimated based on age and for subjects with high thoracic lesions, the lesion level was taken into account. This range is divided into 3 equal parts, resulting in 4 increasing levels. A similar division was made for the stimulation intensity. The minimum level (minimal contraction, MC), was the lowest intensity at which the subject's muscle showed visible contraction, assessed by the investigator. Maximum intensity was $145 \mathrm{~mA}$ (100\%). When heart rate increased to the first predetermined level, consequently, stimulation intensity was adjusted to the first level. There were four levels of stimulation intensity: MC, 33, 66, and 100\%. Increase of the stimulation intensity accompanied the increase of a power level (i.e. at a full minute). The goal of this protocol is to exhaust the arm and leg muscles simultaneously. The endpoint of the test is determined as the moment when cadence fell below 35 RPM and when power output dropped below $70 \%$ of the imposed power.

During the GHT, peak oxygen consumption (VO2peak) and expired ventilation (VE) were sampled every 30 seconds using an automated gas analyzing system (Oxycon Delta, Jaeger, Hoechberg, Germany). Heart rate (HR) was recorded with an interval of $5 \mathrm{sec}-$ onds using a Polar sport tester. Power output (PO) was measured using the computer software of the treadmill (Tacx, Wassenaar, The Netherlands). Values for VO2peak, VEpeak and POpeak were analyzed during the last full minute of cycling, as a mean of two 30second blocks. When the subject completed a full resistance level (a whole 1-minute block) at the stopping moment, this level was taken as POpeak. However when the subject did not complete a block but stopped cycling within a block after at least 30 seconds, POpeak was calculated by averaging the resistance of this block and the resistance of the last completed block. When the subject stopped cycling before completing half of the block, the resistance level of the last completed block was taken as POpeak. Before and after the test, blood pressure was measured manually for safety reasons.

\subsubsection{Power measurement of the legs}

During the GHT the power of the legs is measured using force transducers (O-tec, Lorsch, Germany), which are installed on the pedals. These force transducers 
measure effective (perpendicular on the crank) and ineffective forces applied on the pedals for each leg. The resultant forces and the power output of the legs can be calculated. The power produced by the legs is defined by delta power $(\mathrm{dP})$. The measured power during the first 'warm-up' stage of the GHT, when the legs are not yet actively contributing, is considered as reference value. The variable $\mathrm{dP}$ is the result of a subtraction of this reference value from the mean measured power at the various stimulation intensity levels (MC, 33, 66, and $100 \%$ ).

\subsection{Statistical analysis}

To determine the effect of 4 weeks of hybrid training, pre and post testing was compared using paired two-tailed Students t-tests. To assess the effect in dP, a multi-variate analysis of variance (MANOVA) with repeated measures was used, to check for interactions. A two-tailed $\alpha$ of $<0.05$ was considered to be statistically significant. All data were presented as mean (standard deviation (SD)).

\section{Results}

\subsection{Training results}

All subjects completed the training period. In two subjects heart rate data were missing due to technical problems. Mean training frequency for all subjects was 2.33 (0.33) training sessions per week. Mean duration was 33.7 (2.9) minutes. Mean heart rate during the training was $120(6.9) \operatorname{BPM}(n=8)$.

\subsection{Measurement results}

\subsubsection{Leg volume}

Mean upper leg volume of all subjects increased from $4.7(0.6)$ to $5.1(0.7)$ liters $(p=0.047)$ for the right and from $4.7(0.6)$ to $5.1(0.9)$ liters $(p=0.018)$ for the left upper leg. Mean lower leg volume for all subjects did not change. In Fig. 3 the results are presented for the right leg only. The results of the left leg were in accordance with those of the right leg. There was no change in skin-fold thickness at the quadriceps muscle. It was 15.1 (7.61) vs. $14.3(6.24) \mathrm{mm}$, for pre and post training, respectively.
Table 1

Contrasts in $\mathrm{dP}(\mathrm{ddP})$ between the levels of stimulation intensity pre and post training $(n=9)$

\begin{tabular}{lcc}
\hline $\begin{array}{l}\text { Levels of } \\
\text { stimulation intensity }\end{array}$ & $\begin{array}{c}\text { ddP pre } \\
\text { training (Watt) }\end{array}$ & $\begin{array}{c}\text { ddP post } \\
\text { training (Watt) }\end{array}$ \\
\hline $33 \%-\mathrm{MC}$ & 4.70 & 6.34 \\
$66 \%-33 \%$ & 0.38 & 4.17 \\
$100 \%-66 \%$ & 0.66 & 4.36 \\
\hline
\end{tabular}

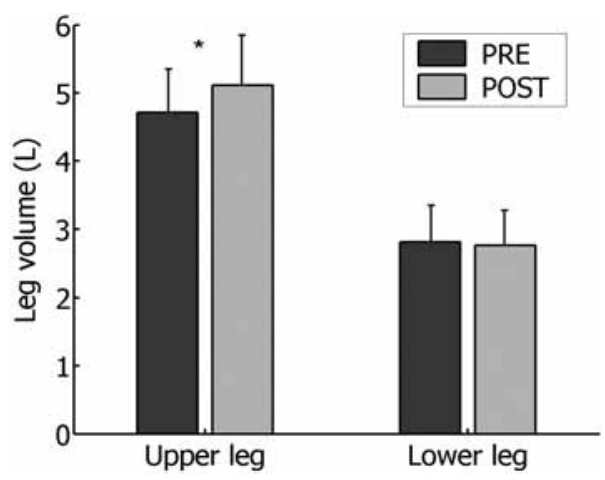

Fig. 3. Mean leg volume for the right upper and lower leg, pre and post training. Error bars represent standard deviation. ${ }^{*} p<0.05$.

\subsubsection{Graded hybrid exercise test}

After the training period, subjects increased their VO2peak ( $p=0.015)$, and POpeak $(p=0.012)$. VO2peak increased $9.3 \%$ (from 25.7 (5.8) to 28.1 (7.5) $\mathrm{ml} / \mathrm{min} / \mathrm{kg}$ ) (Fig. 4A); POpeak increased $11.7 \%$ (from 85.5 (22.5) to 95.5 (25.8) Watt) (Fig. 4C). No significant change was found for VEpeak (Fig. 4B).

\subsection{Power measurements of the legs}

Differences in power produced by the legs compared to passive cycling during GHT are presented in Fig. 5. Multivariate testing showed no significant difference in $\mathrm{dP}$ between pre and post training taken over all stimulation levels (MC, 33\%, 66\%, and $100 \%)(F(1,8)=0.313$; NS). When observing Fig. 5, pre-training measurement shows an even course for $33 \%-100 \%$, whereas post-training measurement shows an increase for the same period. A significant measurement by stimulation interaction was found $(F(3,6)=11.698 ; p=0.006)$, which supports this observation. Table 1 clarifies this interaction. Although stimulation intensity is increased, there is very little additional power produced during the pre-training measurement. In contrast, during the post-training measurement, every step of increased stimulation intensity leads to increased dP. Post-hoc comparisons failed to show significant contrasts between any of the levels. 

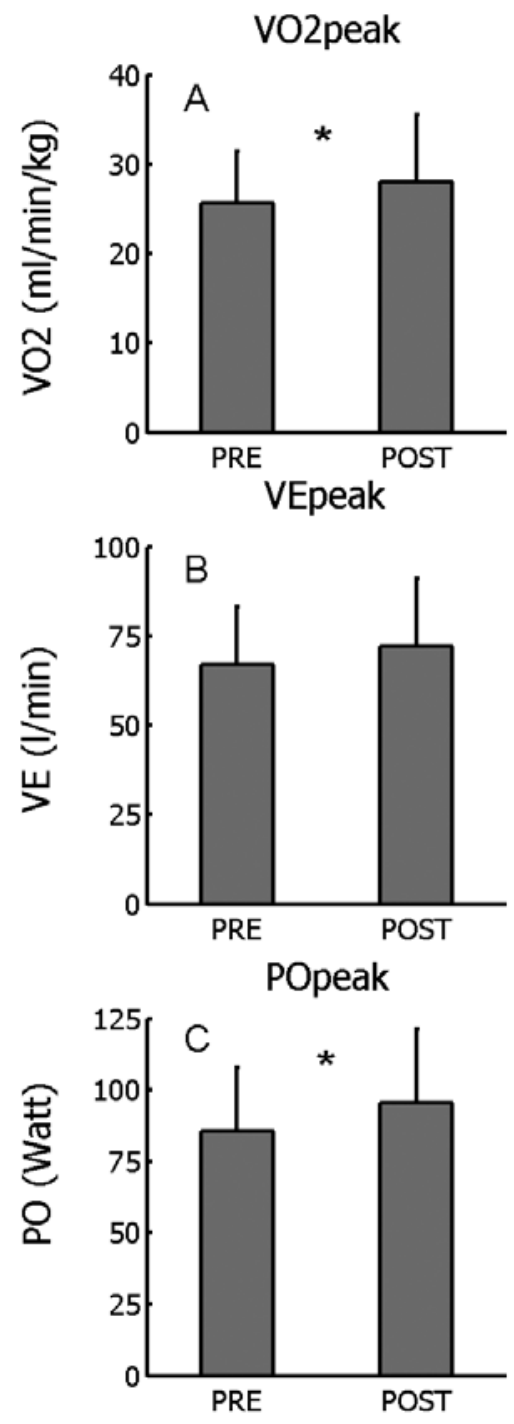

Fig. 4. Peak values during the GHT. A) Mean VO2peak, B) Mean VEpeak, and C) Mean POpeak, all pre and post training $(n=10)$. Error bars represent standard deviation. $* p<0.05$.

\section{Discussion}

The purpose of this study was to determine the effects of hybrid training on a newly developed hybrid FES-cycle on physical fitness in spinal cord-injured individuals. In this study several beneficial and clinically important effects on physical fitness were found. Although these results were not experimentally compared to other training methods, this study shows interesting results which may lead to more comparative studies and field implementation.

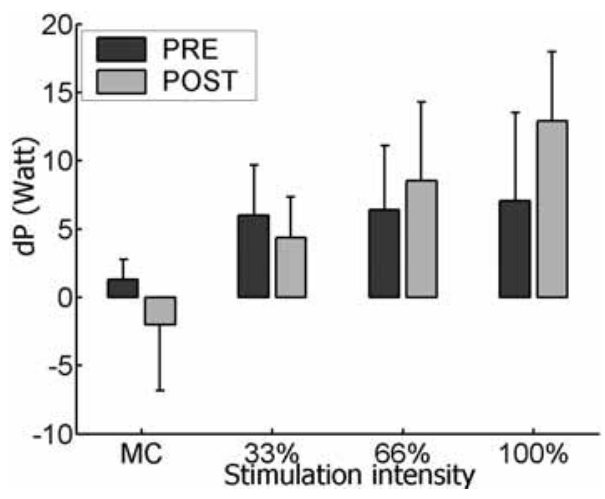

Fig. 5. Mean differences in power compared with passive cycling (dP) of both legs for various levels of stimulation intensity pre and post training $(n=10)$. Error bars represent standard deviation. No significant difference between pre and post training was found, but there was found a significant measurement by stimulation interaction.

\subsection{Leg volume}

The increase in upper leg volume as found in the present study can be explained by training on the hybrid FES-cycle. Subjects performed no other activities with their legs. Unchanged lower leg volume and unchanged skin-fold above the quadriceps give rise to the hypotheses that the increase in upper leg volume is caused by hypertrophy of the thigh muscles following electrical stimulated training. In literature no other studies were found which used the present method, hence comparisons are difficult to make. Sköld et al. reported an increase of about $10 \%$ in muscle tissue volume in the lower extremity measured by CT after six months of three times per week 30 minute FEScycling [28]. Since the duration of training was more than six times shorter in the present study, this may emphasize the early onset of training effects following hybrid FES-cycling.

\subsection{Graded hybrid exercise test}

The increased peak oxygen uptake (VO2peak) was probably due to the larger active muscle mass and therefore greater metabolic rates. VO2peak is an appropriate measure for physical fitness and is sensitive to changes [32]. In the literature no reference values were found for physical fitness following hybrid training with FES on a tricycle but some data are available from other training methods. Janssen et al. (2002) presented normative values for a large group of SCI individuals. Peak VO2 was measured during a graded wheelchair ergometer test and values were 22.8 (8.9) and 24.7 (9.7) $\mathrm{ml} / \mathrm{min} / \mathrm{kg}$ for Th1-Th5 and Th6-Th10, 
respectively [12]. Mean VO2peak for all subjects in the present study was $25.7(5.8) \mathrm{ml} / \mathrm{min} / \mathrm{kg}$ pre training, which indicates that the subjects had fitness levels corresponding to the population of Janssen et al., Mutton et al. reported pre hybrid training VO2peak of $1.69(0.64) \mathrm{l} / \mathrm{min}$ and post hybrid training a VO2peak of $1.91(0.49) 1 / \mathrm{min}$ [18]. The corresponding values in the present study were $1.87(0.52)$ and $2.04(0.58)$ pre and post training, respectively, which was slightly higher. Although the training method in the study of Mutton et al. was different (i.e. they used separate devices for FES-LCE and arm crank exercise simultaneously) the results of the present study indicate similar training effects although the number of training sessions was smaller in the present study (i.e. 10 vs. 4130 -minute sessions for the present study and the study of Mutton et al., respectively) [18].

Peak power output (POpeak) increased remarkably. Compared to Raymond et al., peak PO was high, even slightly higher at baseline. Peak PO in the study of Raymond was 79.5 (6.3) W for hybrid exercise in untrained subjects compared to $85.5(22.5) \mathrm{W}$ pre training in the present study [24].

\subsection{GHT protocol}

In the present GHT protocol, stimulation intensity was increased based on the subject's heart rate. Limitation of this protocol is that heart rate decides the increase of stimulation intensity. If the heart rate of the subject only minimally increases and does not reach the calculated threshold value for the highest stimulation value during the test, the stimulation intensity can not reach maximal stimulation of the legs. During the post-training measurement, a decreased heart rate was observed in most subjects, directly after increasing the stimulation intensity. A possible explanation for this might be the enhanced active muscle mass which serves as a muscle pump, increasing the venous return to the heart. Consequently the protocol does not fit to the subject's exhaustion, and the highest stimulation intensity might not be reached due to the decreased (and more efficient) heart rate. However, when this phenomenon was observed, the stimulation intensity was set at the highest level to be sure that the leg muscles of all subjects received maximal stimulation at the end stage of each GHT.

\subsection{Power measurements of the legs}

A higher stimulation intensity did not lead to a higher power output by the legs (dP) during the pre-training measurement. This can be explained by the untrained state of the leg muscles. The muscles were already exhausted after $33 \%$ stimulation intensity (see Fig. 5 and Table 1) and therefore were not able to significantly contribute to the cycling exercise during the remaining part of the test. In contrast, during the post-training measurement, a higher stimulation intensity leads to a higher power. This can be explained by increased endurance of the leg muscles after training. The leg muscles are strong enough to maintain active contraction and thereby contributing to cycling.

There are no studies available with coupled arm and leg exercise systems. Other hybrid training studies used a FES-cycling system along with a separate arm crank ergometer [18,25]. The present FES-cycle is perfectly suitable for hybrid training, but the measurement of the power of the legs (dP) on this system has its limitations. The power measurement device was installed on the pedals. Since the pedals are propelled by the legs as well as by the arms and because the arm muscles in spinal cord-injured individuals are more powerful than the (trained as well as untrained) leg muscles, our measured values of $\mathrm{dP}$ are probably strongly influenced by arm forces acting on the pedals. In future experiments it would therefore be of interest to additionally measure the forces acting on the hand pedals as well.

\section{Conclusions}

In summary, in this study it was possible to show improvement of physical fitness after a 4-week hybrid training on the hybrid FES-cycle. These results show that this training method is a valid addition to the usual rehabilitation for the spinal cord-injured population.

\section{Acknowledgement}

The authors want to acknowledge Berbke van Ginneken for her assistance in training the subjects and collecting data during measurements and Martijn van der Eijk for his assistance in developing the GHT in their trainee periods, Göran Zemack for performing medical screenings, Hennie Rijken for assisting during measurement sessions, Bart Nienhuis and Brenda Groen for technical and statistical support and the subjects for participating in the study. This study was subsidized by the Sint Maartenskliniek and by ESKAN (Economic Stimulation Junction Arnhem-Nijmegen). 


\section{References}

[1] P.D. Chilibeck, J. Jeon, C. Weiss, G. Bell and R. Burnham, Histochemical changes in muscle of individuals with spinal cord injury following functional electrical stimulated exercise training, Spinal Cord 37 (1999), 264-268.

[2] S.F. Figoni, R.M. Glaser, M.M. Rodgers, B.N. Ezenwa, S.P. Hooker, P.D. Faghri and S.C. Gupta, Hemodynamic responses of quadriplegics to arm, ES-leg and combined arm+ES-leg ergometry, Med. Sci. Sports Exerc. 21 (1989), S96.

[3] H.M. Franken, P.H. Veltink, M.J. IJzerman, F.S. Withaar and H.B.K. Boom, The influence of voluntary upper body exercise on the performance of stimulated paralysed human quadriceps, J. Electromyogr. Kinesiol. 7 (1997), 67-77.

[4] H.L. Gerrits, A. De Haan, M.T. Hopman, L.H. Der Woude, D.A. Jones and A.J. Sargeant, Contractile properties of the quadriceps muscle in individuals with spinal cord injury, Muscle Nerve 22 (1999), 1249-1256.

[5] H.L. Gerrits, A. De Haan, A.J. Sargeant, A. Dallmeijer and M.T. Hopman, Altered contractile properties of the quadriceps muscle in people with spinal cord injury following functional electrical stimulated cycle training, Spinal Cord 38 (2000), 214-223.

[6] H.L. Gerrits, A. De Haan, A.J. Sargeant, H. van Langen and M.T. Hopman, Peripheral vascular changes after electrically stimulated cycle training in people with spinal cord injury, Arch. Phys. Med. Rehabil. 82 (2001), 832-839.

[7] M. Gfohler, M. Loicht and P. Lugner, Exercise tricycle for paraplegics, Medical \& Biological Engineering \& Computing 36 (1998), 118-121.

[8] R.M. Glaser, Functional neuromuscular stimulation. Exercise conditioning of spinal cord injured patients, Int. J. Sports Med. 15 (1994), 142-148.

[9] N. Hjeltnes, A.K. Aksnes, K.I. Birkeland, J. Johansen, A. Lannem and $\mathrm{H}$. Wallberg-Henriksson, Improved body composition after $8 \mathrm{wk}$ of electrically stimulated leg cycling in tetraplegic patients, Am. J. Physiol 273 (1997), R1072-R1079.

[10] S.P. Hooker, S.F. Figoni, M.M. Rodgers, R.M. Glaser, T. Mathews, A.G. Suryaprasad and S.C. Gupta, Metabolic and hemodynamic responses to concurrent voluntary arm crank and electrical stimulation leg cycle exercise in quadriplegics, J. Rehabil. Res. Dev. 29(3) (1992), 1-11.

[11] M.T. Hopman, J.T. Groothuis, M. Flendrie, K.H. Gerrits and S. Houtman, Increased vascular resistance in paralyzed legs after spinal cord injury is reversible by training, J. Appl. Physiol. 93 (2002), 1966-1972.

[12] T.W. Janssen, A.J. Dallmeijer, D.J. Veeger and L.H. van der Woude, Normative values and determinants of physical capacity in individuals with spinal cord injury, J. Rehabil. Res. Dev. 39 (2002), 29-39.

[13] T.W. Janssen, R.M. Glaser and D.B. Shuster, Clinical efficacy of electrical stimulation exercise training: effects on health, fitness, and function, Topics in Spinal Cord Injury Rehabilitation 3 (1998), 33-49.

[14] T.W.J. Janssen and R.M. Glaser, Electrical stimulationinduced leg cycling in individuals with spinal cord injury, in: Biomedical Aspects of Manual Wheelchair Propulsion, L.H.V. Woude van der et al., eds, IOS Press, 1999, pp. 251-262.

[15] P.R. Jones and J. Pearson, Anthropometric determination of leg fat and muscle plus bone volumes in young male and female adults, J. Physiol. 204 (1969), 63P-66P.
[16] M. Kjaer, S.F. Pollack, T. Mohr, H. Weiss, G.W. Gleim, F.W. Bach et al., Regulation of glucose turnover and hormonal responses during electrical cycling in tetraplegic humans, Am. J. Physiol. 271 (1996), R191-R199.

[17] T. Mohr, J.L. Andersen, F. Biering-Sorensen, H. Galbo, J. Bangsbo, A. Wagner et al., Long-term adaptation to electrically induced cycle training in severe spinal cord injured individuals, Spinal Cord 35 (1997), 1-16.

[18] D.L. Mutton, A.M. Scremin, T.J. Barstow, M.D. Scott, C.F. Kunkel and T.G. Cagle, Physiologic responses during functional electrical stimulation leg cycling and hybrid exercise in spinal cord injured subjects, Arch. Phys. Med. Rehabil. (1997), 712-718.

[19] J.S. Petrofsky, Functional electrical stimulation, a two-year study, Journal of Rehabilitation (1992), 29-34.

[20] J.S. Petrofsky, H. Heaton, III and C.A. Phillips, Outdoor bicycle for exercise in paraplegics and quadriplegics, J. Biomed. Eng 5 (1983), 292-296.

[21] J.S. Petrofsky and J. Smith, Three-wheel cycle ergometer for use by men and women with paralysis, Med. Biol. Eng Comput. 30 (1992), 364-369.

[22] D.J. Pons, C.L. Vaughan and G.G. Jaros, Cycling device powered by the electrically stimulated muscles of paraplegics, Med. Biol. Eng. Comput. 27 (1989), 1-7.

[23] J. Quintern, Application of functional electrical stimulation in paraplegic patients, NeuroRehabilitation 10 (1998), 205-250.

[24] J. Raymond, G.M. Davis, M. Climstein and J.R. Sutton, Cardiorespiratory responses to arm cranking and electrical stimulation leg cycling in people with paraplegia, Med. Sci. Sports Exerc. 31(6) (1999), 822-828.

[25] J. Raymond, G.M. Davis, A. Fahey, M. Climstein and J.R. Sutton, Oxygen uptake and heart rate responses during arm vs combined arm/electrically stimulated leg exercise in people with paraplegia, Spinal Cord 35 (1997), 680-685.

[26] A.M. Scremin, L. Kurta, A. Gentili, B. Wiseman, K. Perell, C. Kunkel et al., Increasing muscle mass in spinal cord injured persons with a functional electrical stimulation exercise program, Arch. Phys. Med. Rehabil. 80 (1999), 1531-1536.

[27] M.L. Sipski, J.A. DeLisa and S. Schweer, Functional electrical stimulation bicycle ergometry: patient perceptions, Am. J. Phys. Med. Rehabil. 68 (1989), 147-149.

[28] C. Sköld, L. Löhn, K. Harms-Ringdahl, C. Hultling, R. Levi, M. Nash et al., Effects of functional electrical stimulation training for six months on body composition and spasticity in motor complete tetraplegic spinal cord-injured individuals, $J$. Rehabil. Med. 34 (2002), 25-32.

[29] R. Stein, Functional electrical stimulation after spinal cord injury, Journal of Neurotrauma 16 (1999), 713-717.

[30] R.B. Stein, S.L. Chong, K.B. James and G.J. Bell, Improved efficiency with a wheelchair propelled by the legs using voluntary activity or electric stimulation, Arch. Phys. Med. Rehabil. 82 (2001), 1198-1203.

[31] R.B. Stein, D. Roetenberg, S.L. Chong and K.B. James, A wheelchair modified for leg propulsion using voluntary activity or electrical stimulation, Med. Eng. Phys. 25 (2003), 11-19.

[32] M.W. Stewart, S.L. Melton-Rogers, S. Morrison and S.F. Figoni, The measurement properties of fitness measures and health status for persons with spinal cord injuries, Arch. Phys. Med. Rehabil. 81 (2000), 394-400. 